\title{
Construção e validação de um vídeo educativo sobre a reflexologia podal
}

\author{
Natiele Favarão da Silva ${ }^{1}$, Natália Chantal Magalhães da Silva², Vanessa dos Santos Ribeiro ${ }^{3}$, \\ Denise Hollanda Iunes ${ }^{4}$, Emília Campos de Carvalho ${ }^{5}$
}

\author{
${ }^{1}$ Enfermeira. Residente do Hospital de \\ Reabilitação de Anomalias Craniofaciais da \\ Universidade de São Paulo. Bauru, SP, \\ Brasil. E-mail: natiele.silva@usp.br. \\ ${ }^{2}$ Enfermeira, Doutora em Ciências. \\ Professora Adjunta do Centro Universitário \\ de Belo Horizonte. Belo Horizonte, MG, \\ Brasil. E-mail: nchantal@usp.br. \\ ${ }^{3}$ Enfermeira. Discente do Programa de \\ Pós-Graduação em Enfermagem \\ Fundamental, nível Mestrado, da Escola de \\ Enfermagem de Ribeirão Preto da \\ Universidade de São Paulo. Ribeirão Preto, \\ SP, Brasil. E-mail: \\ vanessa.santos.ribeiro@usp.br. \\ ${ }^{4}$ Fisioterapeuta, Doutora em Ciências. \\ Professor Adjunto da Universidade Federal \\ de Alfenas. Alfenas, MG, Brasil. E-mail: \\ deniseiunes@unifal-mg.edu.br. \\ ${ }^{5}$ Enfermeira, Doutora em Ciências. \\ Professora Sênior da Escola de \\ Enfermagem de Ribeirão Preto da \\ Universidade de São Paulo. Ribeirão Preto, \\ SP, Brasil. E-mail: ecdcava@usp.br.
}

Recebido: 28/11/2016.

Aceito: 13/09/2017.

Publicado: 31/12/2017.

\section{Como citar esse artigo:}

Silva NF, Silva NCM, Ribeiro VS, lunes DH, Carvalho EC. Construção e validação de um vídeo educativo sobre a reflexologia podal. Rev. Eletr. Enf. [Internet]. 2017 [acesso em: ____ ] 19:a48. Disponível em: http://doi.org/10.5216/ree.v19.44324.

\begin{abstract}
RESUMO
Este estudo teve como objetivo construir e validar um vídeo educativo sobre a reflexologia podal. Estudo metodológico, desenvolvido em uma Instituição de Ensino Superior do sudeste brasileiro, em que foram percorridas as fases de pré-produção, produção e pós-produção do vídeo, seguidas da avaliação da compreensão e da abrangência do conteúdo abordado. A versão final do vídeo educativo apresenta duração de 12 minutos e sete segundos. Os peritos o avaliaram como um recurso educativo que apresenta a temática de forma clara e objetiva. Os alunos consideraram o material educativo adequado e apontaram boa aceitação do mesmo. As etapas adotadas permitiram construir e validar o vídeo proposto, um material educativo claro, objetivo e adequado. Espera-se, em estudo posterior, avaliar o impacto do vídeo educativo na construção do conhecimento da reflexologia podal.

Descritores: Filmes e Vídeos Educativos; Massagem; Educação em Saúde; Cuidados de Enfermagem.
\end{abstract}

\section{INTRODUÇÃO}

Teve a finalidade de incentivar a utilização de novas estratégias de assistência, de políticas e de resoluções que propõem a adoção de práticas integrativas e complementares na área da saúde ${ }^{(1)}$. Dentre essas práticas, destaca-se a reflexologia podal, que visa o equilíbrio do organismo a partir da pressão em pontos específicos dos pés ${ }^{(2)}$.

Considerada uma terapia simples e de fácil aplicação, pesquisas envolvendo a utilização da reflexologia podal têm sido incentivadas; seus resultados indicam que esta prática pode aumentar a disponibilidade de estratégias de cuidado e, consequentemente, a melhoria da qualidade da assistência ${ }^{(1-3)}$.

Nesse contexto e frente à visão de integralidade requerida aos profissionais de saúde, alguns 
pesquisadores incentivam a abordagem das práticas integrativas e complementares nos cursos da área da saúde, tendo em vista a formação holística do profissional e o aumento da disponibilidade de estratégias de cuidado $^{(4)}$. Além disso, tendências nesse campo podem trazer implicações para o relacionamento entre profissional e paciente e para o futuro do cuidado holístico ${ }^{(2)}$.

Destaca-se a ausência de discussões e a insuficiência de estudos que fundamentem a metodologia de ensino de tais práticas ${ }^{(5)}$. A abordagem da temática em nosso meio, quando presente, ocorre de forma esporádica por meio de palestras, de seminários e de disciplinas optativas, o que restringe o aprofundamento do conhecimento dos profissionais. Assim, a adoção de estratégias inovadoras devem ser implementadas para facilitar o ensino e auxiliar a consolidação do aprendizado ${ }^{(4)}$.

Frente à necessidade de fornecer aos estudantes da área da saúde conhecimentos para a implementação da reflexologia podal, justifica-se o desenvolvimento de um vídeo educativo na área. Assim, esta proposta teve como objetivo construir e validar um vídeo educativo sobre a reflexologia podal.

\section{MÉTODO}

Estudo metodológico, com delineamento longitudinal e análise descritiva ${ }^{(6)}$, realizado em uma Instituição de Ensino Superior do sudeste brasileiro, de janeiro de 2015 a agosto de 2016.

Foram percorridas as fases de pré-produção, de produção e de pós-produção do vídeo ${ }^{(7)}$, seguidas da avaliação da compreensão e da abrangência do conteúdo abordado.

\section{Pré-produção}

A fase de pré-produção baseou-se em três etapas: construção do roteiro do vídeo, elaboração do storyboard e validação deste por peritos ${ }^{(7)}$.

Inicialmente, o roteiro foi construído a partir do conhecimento dos autores e dos achados de uma revisão integrativa, realizada durante os meses de janeiro e fevereiro de 2015, a partir da questão norteadora "Qual o conhecimento produzido sobre a reflexologia podal?". A busca foi realizada nas bases de dados CINAHL, PUBMED, SCIENCE DIRECT e WEB OF SCIENCE, utilizando-se a palavra-chave "foot reflexology".

Com a finalidade de orientar o processo de criação, o roteiro foi construído em forma de um quadro, com duas colunas. Na primeira coluna, foi descrito o conteúdo correspondente a cada cena futura e, na segunda, os personagens e/ou recurso audiovisual a ser utilizado (como imagens, cenas, animações, textos, narração e sons de fundo), o que deu origem ao storyboard do vídeo. Este, composto por 45 cenas, foi encaminhado para a análise de cinco peritos com experiência na área da reflexologia podal.

Para a análise do storyboard pelos peritos, foi elaborado um instrumento adaptado pelos critérios propostos por López ${ }^{(8)}$, com itens relacionados à congruência do conteúdo e à pertinência da inclusão dos tópicos do roteiro. 


\section{Produção}

A produção, segunda fase do estudo, consistiu na implementação das ideias elaboradas na préprodução (storyboard) e foi dividida em quatro etapas: ensaio das cenas com os atores; filmagem; narração e desenvolvimento de imagens e animações ${ }^{(7)}$.

Após o ensaio dos atores e a realização dos ajustes pertinentes para o alcance de boa qualidade técnica, ocorreram a filmagem e a narração a ser inserida no vídeo; para essa etapa, utilizou-se laboratório que dispunha de estrutura física e de recursos tecnológicos para a gravação. Nessa etapa, visando à iluminação e à angulação adequadas que proporcionassem imagens com boa qualidade, contou-se com o auxílio de uma equipe técnica especializada em recursos audiovisuais.

Dois autores representaram os papéis de enfermeira e de paciente, para a demonstração do procedimento durante as filmagens. Já a narração inserida do vídeo, foi realizada de forma voluntária por uma funcionária da Instituição de Ensino onde se deu o estudo e ocorreu em ambiente que apresentava condições acústicas favoráveis para a gravação do áudio.

Para o desenvolvimento das imagens e das animações, foi observada a legislação referente aos direitos autorais de utilização e de reprodução de $\operatorname{recursos}^{(9)}$. Dessa forma, a captura de imagens de terceiros se deu pelo site Creative Commons, organização sem fins lucrativos que permite o compartilhamento e o uso da criatividade e do conhecimento por meio de instrumentos jurídicos gratuitos ${ }^{(10)}$. As demais imagens e animações do vídeo foram desenvolvidas a partir do programa Adobe Illustrator ${ }^{\circledast}$ e Adobe Flash ${ }^{\circledR}$.

\section{Pós-produção}

A terceira fase do estudo, pós-produção, contemplou duas etapas: edição e validação do vídeo por peritos $^{(7)}$.

$\mathrm{Na}$ edição, foram realizados ajustes nas gravações das cenas e da narração, com a inclusão de imagens, de textos e de animações ao vídeo. Para tanto, foram utilizados os programas Adobe Premiere ${ }^{\circledR}$ e Adobe Audacity ${ }^{\circledR}$.

Após a edição, o conteúdo do vídeo (narração, imagens, animações e filmagem) foi transferido para o formato DVD (Digital Versatile Disk) por meio do programa Avid Liquid Pro ${ }^{\circledR}$, versão sete; foi então encaminhado a cinco peritos com experiência em recursos educativos em saúde. Estes avaliaram o material educativo, a partir de um instrumento adaptado pelos critérios propostos por López ${ }^{(8)}$. A validação consistiu no apontado, pelos peritos, da existência do conteúdo especificado no instrumento no vídeo e se essa inserção era desejável ao vídeo. Também avaliou-se o ambiente, a atuação dos personagens e a execução do procedimento em estudo.

\section{Avaliação da compreensão e da abrangência do conteúdo abordado}

Com a finalidade de verificar a compreensão e a abrangência do conteúdo abordado, estudantes da área da saúde avaliaram o vídeo. 
Como critérios de inclusão para participação, foram adotados: estar regularmente matriculado na Instituição de Ensino onde foi realizado o estudo e ter obtido aprovação na disciplina de anatomia, visto que a técnica que envolve a aplicação da reflexologia podal requer o conhecimento de áreas e de regiões dos pés.

O recrutamento foi do tipo "bola de neve", que consiste na seleção de participantes por meio da indicação de um participante anterior ${ }^{(6)}$. Dessa forma, um estudante escolhido ao acaso, após ter sido avaliado quanto aos critérios de elegibilidade para participação do estudo, assistiu ao vídeo e procedeu a avaliação deste. Em seguida, foi solicitado que o estudante indicasse outro e, assim, sucessivamente, até o alcance de 10 estudantes.

A avaliação da compreensão e da abrangência baseou-se no estudo de Braga ${ }^{(11)}$. Assim, após assistirem ao vídeo, os estudantes atribuíram um valor, em uma escala de zero a 10, a cada tópico abordado - conceito das práticas integrativas e complementares; utilização das práticas integrativas e complementares; e, com relação à reflexologia podal: conceito e histórico, suas finalidades, situações em que não é indicada, localização dos pontos reflexos nos pés, preparação para aplicação e técnicas de uso.

De modo complementar, os estudantes avaliaram a qualidade do material audiovisual e a quantidade de vezes que seria necessário assistir ao vídeo para a aquisição de informações.

\section{Aspectos éticos}

Para assegurar os direitos dos participantes e cumprir os aspectos contidos na Resolução 466 do Ministério da Saúde ${ }^{(12)}$, esta proposta foi submetida ao Comitê de Ética em Pesquisa envolvendo seres humanos da Escola de Enfermagem de Ribeirão Preto (EERP-USP), sob o parecer no 1.196.372. Ademais, os atores do vídeo assinaram o Termo de Autorização de Uso da Imagem; a narradora, o Termo de Autorização de Uso de Voz; e os peritos e os estudantes, o Termo de Consentimento Livre e Esclarecido - TCLE, em duas vias.

\section{RESULTADOS}

A construção e a validação do vídeo educativo, ao percorrer as fases de pré-produção, da produção, da pós-produção e da avaliação da compreensão e da abrangência do conteúdo abordado, compreendeu 18 meses, com início em janeiro de 2015 e término em julho de 2016.

As sugestões dos peritos e dos estudantes que participaram do estudo foram discutidas entre os autores e acatadas, quando pertinentes.

\section{Pré-produção}

Após a revisão integrativa da literatura, foram extraídas informações de 27 artigos e de dois livros para a construção do roteiro. Esse foi desmembrado em 41 cenas e compôs o storyboard do vídeo.

Cinco peritos participaram da validação do storyboard. Todos eram do sexo feminino, sendo quatro 
fisioterapeutas e uma enfermeira, com idade média de 40,8 anos (DP = 4,8); tempo de formação médio de 18,2 anos ( $D P=7,1$ ) e tempo de atuação profissional médio de 18,2 anos ( $D P=7,07$ ). Na área da reflexologia podal, os peritos apresentaram tempo de atuação médio de 8,6 anos (DP = 5,6) e todos referiram publicações relacionadas à terapia.

$\mathrm{Na}$ avaliação da congruência do conteúdo, todos os peritos consideraram as informações do storyboard compreensíveis, com sequência lógica e com linguagem de fácil assimilação, adequada ao público alvo.

No tocante à pertinência da inclusão dos tópicos, os cinco peritos referiram que o conceito e a utilização das práticas integrativas e complementares foram apresentadas. Nos itens relacionados à reflexologia podal - finalidade; situações em que não é indicada; surgimento; localização dos pontos; preparação e técnica de aplicação - todos alegaram que estavam contidos no storyboard; contudo, um apontou que a definição da terapia não estava clara, o que levou à realização de ajustes neste item.

\section{Produção}

Após a validação do storyboard pelos peritos, foram realizados ensaios com os atores, que totalizaram quatro horas de duração.

A filmagem das cenas e a narração do vídeo ocorreram em dois dias consecutivos. No primeiro dia, após finalizada a filmagem e a narração, os autores se reuniram e verificaram a necessidade de certos ajustes na iluminação e na acústica. Assim, no dia seguinte, optou-se pela realização de nova filmagem e de narração do vídeo.

No que se refere ao desenvolvimento de imagens e de animações, foram capturadas e incluídas no vídeo 14 imagens de terceiros e criadas 26 imagens/animações.

\section{Pós-produção}

Com a finalidade de melhorar a qualidade do vídeo, foram realizadas reuniões periódicas com a equipe técnica especializada em recursos audiovisuais, totalizando 16 horas destinadas à edição do vídeo.

Assim, após as edições necessárias, o vídeo foi encaminhado para a validação de peritos.

Os cinco peritos que participaram da validação do vídeo eram do sexo feminino e apresentavam idade média de 39,4 anos ( $D P=1,41)$. Quanto à formação profissional, três eram enfermeiras e duas fisioterapeutas, com média de tempo de formação e de atuação profissional de 16,8 anos (DP $=1,41)$. Com relação ao tempo de atuação em recursos educativos em saúde, os peritos apresentaram média de 6,6 anos ( $D P=1,41)$ e, a maioria (três peritos), informou publicações relacionadas à terapia.

A Tabela 1 apresenta os itens avaliados pelos peritos na validação do vídeo educativo. 
Tabela 1: Distribuição dos itens relacionados ao vídeo educativo avaliados como existentes e desejáveis pelos peritos ( $\mathrm{n}=5$ ). Ribeirão Preto, SP, Brasil, 2016.

\begin{tabular}{|c|c|c|}
\hline Itens & $\begin{array}{l}\text { Existente } \\
(n=5)\end{array}$ & $\begin{array}{l}\text { Desejável } \\
(n=5)\end{array}$ \\
\hline \multicolumn{3}{|l|}{ Técnica audiovisual } \\
\hline Identificação inicial do conteúdo que se pretende mostrar & 5 & 5 \\
\hline Iluminação necessária para a observação adequada das cenas & 5 & 5 \\
\hline Som necessário para escutar a voz do narrador & 5 & 5 \\
\hline Possibilita, quando desejado, voltar a qualquer parte das cenas & 4 & 5 \\
\hline \multicolumn{3}{|l|}{ Ambiente } \\
\hline O cenário reflete o cotidiano da prática assistencial de enfermagem & 5 & 5 \\
\hline O ambiente e material para a aplicação da terapia são contemplados & 5 & 5 \\
\hline $\begin{array}{l}\text { A simplificação da realidade no laboratório não interfere na fidelidade da terapia que se pretende } \\
\text { mostrar }\end{array}$ & 5 & 5 \\
\hline \multicolumn{3}{|l|}{ Personagens } \\
\hline A linguagem utilizada é compreensível para o público alvo & 5 & 5 \\
\hline A voz do narrador é clara & 5 & 5 \\
\hline O tom de voz do narrador é adequado & 5 & 5 \\
\hline \multicolumn{3}{|l|}{ Procedimento em estudo: Reflexologia podal } \\
\hline \multicolumn{3}{|l|}{ O conteúdo apresentado permite a compreensão dos itens: } \\
\hline Conceito das práticas integrativas e complementares & 5 & 5 \\
\hline Utilização das práticas integrativas e complementares & 5 & 5 \\
\hline Conceito da reflexologia podal & 5 & 5 \\
\hline Finalidade da reflexologia podal & 5 & 5 \\
\hline Situações em que a reflexologia podal não é indicada & 5 & 5 \\
\hline Surgimento da reflexologia podal & 5 & 5 \\
\hline Localização dos pontos reflexos nos pés & 5 & 5 \\
\hline Preparação para a aplicação da reflexologia podal & 5 & 5 \\
\hline Técnicas de reflexologia podal & 5 & 5 \\
\hline Todas as técnicas de aplicação são apresentadas & 5 & 5 \\
\hline As etapas da aplicação da terapia estão adequadas e podem ser identificadas & 5 & 5 \\
\hline O vídeo baseia-se em conteúdo publicado na literatura & 5 & 5 \\
\hline
\end{tabular}

Diante da concordância entre peritos que procederam à avaliação do vídeo, podemos considerar que a técnica audiovisual, o ambiente, os personagens e o procedimento em estudo mostraram-se adequados e apropriados para a população alvo.

Finalizada a validação por peritos e realizados os ajustes necessários, a versão final do vídeo teve duração de 12 minutos e sete segundos.

A Figura 1 apresenta algumas cenas contidas na versão final do vídeo educativo. 




Cena 31
Cena 19

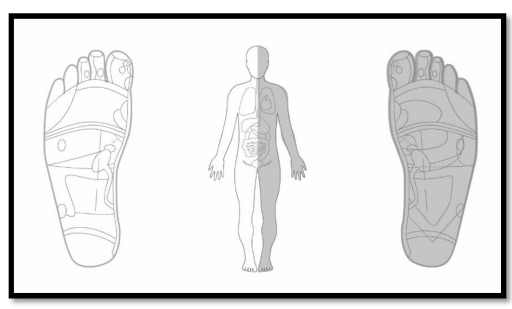

Cena 32

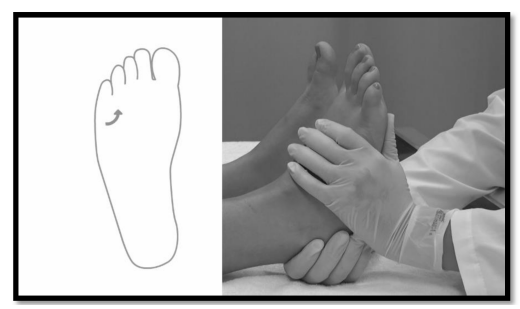

Cena 29

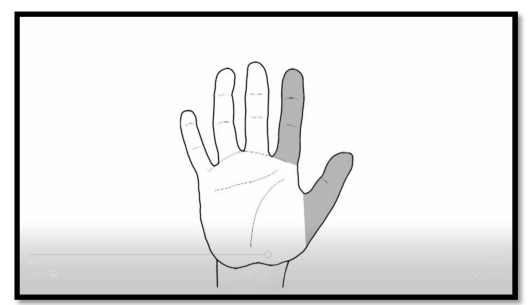

Cena 33

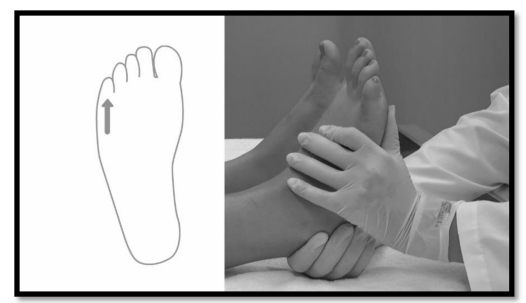

Figura 1: Cenas contidas na versão final do vídeo educativo.

Avaliação da compreensão e da abrangência do conteúdo abordado

A compreensão e a abrangência do conteúdo abordado no vídeo foram avaliadas por 10 estudantes da área da saúde. Estes estavam regularmente matriculados na Instituição de Ensino onde se deu o estudo, sendo que três cursavam o 50 período; um, o 6o período; e seis, o 7으 período do Curso de Enfermagem. A maioria (oito estudantes) era do sexo feminino; com média de 21,9 anos de idade ( $D P=0,71$ ).

Os estudantes atribuíram valores que variaram entre sete e 10, com média de 9,3 ( $D P=0,53$ ), o que evidencia boa compreensão e abrangência do conteúdo do vídeo.

Três estudantes referiram a necessidade de assistir ao vídeo apenas uma vez para a aquisição de conhecimento, enquanto sete alegaram que a apresentação do conteúdo duas vezes proporcionaria melhor apreensão das informações.

Quanto à qualidade do vídeo, foram atribuídos valores entre nove e 10, o que aponta clareza e objetividade do material educativo.

\section{DISCUSSÃO}

Estudos envolvendo a construção e a validação de vídeos educativos na área da saúde ${ }^{(13-19)}$, assim como neste estudo, baseou-se na metodologia proposta por Fleming, Reynolds e Wallace ${ }^{(7)}$, cujos passos contribuem para a construção de um material capaz de informar seu público alvo.

Alguns vídeos educativos foram desenvolvidos com a finalidade de fornecer orientação a pacientes oncológicos em tratamento quimioterápico ${ }^{(11,13)}$; promover o vínculo entre mãe HIV positiva e seu filho ${ }^{(14)}$; orientar a higiene bucal de pacientes em quimioterapia ${ }^{(6)}$; orientar alunos sobre a punção e heparinização de um cateter totalmente implantado ${ }^{(17)}$; orientar a técnica de administração de medicamentos ${ }^{(18)}$; orientar cuidadores de crianças com fissura labiopalatina ${ }^{(19)}$. Não foram encontrados estudos relacionados à construção e validação de vídeos educativos sobre a reflexologia podal.

A utilização de vídeos educativos favorecendo a aquisição do conhecimento e a apreensão da 
informação comprovadas neste estudo também foi verificada por outros autores em outras temáticas ${ }^{(13-19)}$. Estes autores afirmam que a ferramenta apresenta outras vantagens relacionadas ao estimulo para a participação ativa do paciente no processo saúde-doença e para a reintegração destes em suas atividades cotidianas $^{(13-14)}$. Também consiste em uma estratégia benéfica para o processo de ensino-aprendizagem ${ }^{(15-}$ ${ }^{16)}$; se constitui uma ferramenta de apoio educacional que facilita o conhecimento técnico dos estudantes acerca de uma temática abordada ${ }^{(17)}$. Além disso, possibilita maior preparo e confiança para o desempenho dos procedimentos, uma vez que auxilia no autodomínio e na promoção da segurança ${ }^{(18)}$. Outros autores também concordam que há melhor aquisição de conhecimento com a utilização de vídeo, o que reafirma, ainda mais, os benefícios da utilização deste recurso ${ }^{(19)}$.

O vídeo também pode ser utilizado em conjunto com outras estratégias educativas, como é o caso da simulação realística. Em uma pesquisa, por exemplo, situações simuladas foram apresentadas a estudantes por meio de vídeos e, ao se comparar com o ensino didático presencial, os resultados demonstraram melhor desempenho dos estudantes ${ }^{(20)}$. Além das evidências que sugerem os benefícios do emprego do vídeo em atividades de educação, esta ferramenta apresenta elevado custo-benefício ${ }^{(20)}$. Apesar dos gastos relacionados à mão-de-obra especializada e aos recursos audiovisuais necessários para a elaboração da ferramenta, não são necessários gastos com materiais e insumos sempre que o recurso é utilizado, como ocorre em situações simuladas e em treinamento de habilidades práticas.

Quanto à duração do vídeo, é recomendado que este tipo de ferramenta didática não ultrapasse 15 minutos, já que, após este período, a manutenção da atenção de quem o assiste torna-se comprometida ${ }^{(7)}$. Nesse sentido, entende-se que a versão final do vídeo elaborado neste estudo condiz com o que é recomendado na literatura, dado que este apresentou 12 minutos e sete segundos de duração.

No que se refere ao percurso metodológico de construção e validação de um vídeo educativo, considera-se que as etapas de pré-produção e produção são fundamentais para o desenvolvimento de um material de qualidade ${ }^{(11,14)}$. É necessário que o storyboard do vídeo esteja pautado na literatura científica e nas observações de pessoas com real experiência na temática. Ademais, é indispensável a participação de uma equipe técnica, especializada em recursos audiovisuais, para obtenção das cenas do vídeo ${ }^{(11)}$.

Assim, considera-se que a quase nulidade de discordâncias entre os peritos e as evidencias de boa compreensão e abrangência do conteúdo do vídeo produzido podem estar relacionadas ao rigor metodológico percorrido neste estudo.

Pode-se dizer, portanto, que o vídeo educativo sobre a reflexologia podal apresentou-se como uma estratégia educativa de qualidade, o que também foi apontado em estudos que envolveram o desenvolvimento de vídeos como recursos didáticos ${ }^{(13-19)}$.

Visto que o vídeo educativo construído não é uma ferramenta de domínio público, considera-se uma limitação a acessibilidade controlada ao material. 


\section{CONCLUSÃO}

As etapas de pré-produção e produção permitiram construir o vídeo proposto sobre a reflexologia podal. Este, em sua versão final, apresenta 12 minutos e sete segundos de duração.

$\mathrm{Na}$ etapa de pós-produção, os resultados indicaram ausência de discordâncias entre os peritos que procederam a avaliação do vídeo e, de acordo com a avaliação realizada pelos estudantes da área da saúde, o conteúdo do material educativo apresenta boa compreensão e abrangência.

Logo, a partir da análise dos peritos e dos estudantes, tem-que o vídeo introduz a temática da reflexologia podal e apresenta os critérios necessários para a aplicação na população de interesse, tais como clareza e objetividade.

Sugere-se, portanto, a realização de estudo posterior fundamentado na mensuração do impacto do vídeo educativo sobre a reflexologia podal na construção do conhecimento de estudantes da área da saúde.

Assim, dado que os vídeos surgem como uma proposta didática, o material proposto pode atuar como facilitador do processo ensino-aprendizagem de estudantes da área da saúde. O recurso educativo pode ser utilizado para apresentar a temática da reflexologia podal, instigar o estudo e estimular pesquisas na área e, ainda, aumentar as possibilidades de atuação dos futuros profissionais da saúde no campo das práticas integrativas e complementares.

\section{REFERÊNCIAS}

1. Dalal K, Maran VB, Pandey RM, Tripathi M. Determination of Efficacy of Reflexology in Managing Patients with Diabetic Neuropathy: A Randomized Controlled Clinical Trial. Evidence-Based Complement Altern Med [Internet]. 2014 [acesso em: 31 dez. 2017];2014:843036. Disponível em: http://doi.org/10.1155/2014/843036.

2. Bagheri-Nesami M, Shorofi SA, Zargar N, Sohrabi M, Gholipour-Baradari A, Khalilian A. The effects of foot reflexology massage on anxiety in patients following coronary artery bypass graft surgery: a randomized controlled trial. Complement Ther Clin Pract [Internet]. 2014 [acesso em: 31 dez. 2017];20(1):42-7. Disponível em: http://doi.org/10.1016/j.ctcp.2013.10.006.

3. Silva NCM, Chaves ECL, Carvalho EC, Carvalho LC, lunes DH. Foot reflexology in feet impairment of people with type 2 diabetes mellitus: randomized trial. Rev Lat Am Enfermagem [Internet]. 2015 [acesso em: 31 dez. 2017];23(4):603-

10. Disponível em: http://doi.org/10.1590/0104-1169.0036.2594.

4. Castro Sánchez AM, Moreno Lorenzo C, Matarán Peñarrocha GA, Anaya Ojeda J, Sánchez Labraca N, Zurita Ortega F. Disminución de la presión arterial en pacientes con arteriopatía periférica mediante el masaje reflejo del tejido conjuntivo. Fisioterapia [Internet]. 2009 [acesso em: 31 dez. 2017];31(2):50-4. Disponível em: http://doi.org/10.1016/j.ft.2008.06.001.

5. Silva NCM, lunes DH, Resck ZMR, Soares MI, Souza Junior DI, Vieira NF. Estratégias de ensino das terapias alternativas e complementares na graduação em Enfermagem: revisão integrativa. Rev. Eletr. Enf. [Internet]. 2013 [acesso em: 31 dez. 2017];15(4):1061-7. Disponível em: http://doi.org/10.5216/ree.v15i4.20568.

6. Polit DF, Beck CT. Essentials of Nursing Research: Appraising Evidence for Nursing Practice. $8^{\text {th }}$ ed. Oxford: WileyBlackwell; 2013.

7. Fleming SE, Reynolds J, Wallace B. Lights... camera... action! a guide for creating a DVD/video. Nurse Educ [Internet]. 2009 [acesso em: 31 dez. 2017];34(3):118-21. Disponível em: http://doi.org/10.1097/NNE.0b013e3181a0270e.

8. López ML, Carvalho EC. La comunicación terapéutica durante instalación de venoclisis: uso de la simulación filmada. Rev Lat Am Enfermagem [Internet]. 2006 [acesso em: 31 dez. 2017];14(5):658-65. Disponível em: 
http://doi.org/10.1590/S0104-11692006000500004.9. Lei № 9.610, de 19 de fevereiro de 1998 (BR) [Internet]. Altera, atualiza e consolida a legislação sobre direitos autorais e dá outras providências. Diário Oficial da União. 20 fev. 1998 [acesso em: 31 dez. 2017]. Disponível em: http://www.planalto.gov.br/ccivil 03/leis/L9610.htm.

10. Creative Commons Brasil [Internet]. São Paulo: Creative Commons Brasil, c2001-2017 [acesso em: 31 dez. 2017]. Disponível em: https://br.creativecommons.org/.

11. Braga FTMM, Garbin LM, Marmol MT, Khouri VY, Vasques Cl, Carvalho EC. Higiene bucal de pacientes em quimioterapia: construção e validação de um vídeo educativo. Revista de enfermagem UFPE on line [Internet]. 2014 [acesso em: 31 dez. 2017];8(10):3331-9. Disponível em:

https://periodicos.ufpe.br/revistas/revistaenfermagem/article/view/10064.

12. Resolução № 466 do Conselho Nacional de Saúde, de 12 de dezembro de 2012 (BR) [Internet]. Aprova as diretrizes e normas regulamentadoras de pesquisas envolvendo seres humanos. Diário Oficial da União. 12 dez. 2012 [acesso em: 31 dez. 2017]. Disponível em: http://bvsms.saude.gov.br/bvs/saudelegis/cns/2013/res0466_12_12_2012.html. 13. Razera APR, Buetto LS, Lenza NFB, Sonobe HM. Vídeo educativo: estratégia de ensino-aprendizagem para pacientes em tratamento quimioterápico. Cienc Cuid Saude [Internet]. 2014 [acesso em: 31 dez. 2017];13(1):173-8. Disponível em: http://periodicos.uem.br/ojs/index.php/CiencCuidSaude/article/view/19659.

14. Barbosa RM, Bezerra AK. Validação de um vídeo educativo para promoção do apego entre mãe soropositiva para HIV e seu filho. Rev. bras. enferm. [Internet]. 2011 [acesso em: 31 dez. 2017];64(2):328-34. Disponível em:: http://doi.org/10.1590/S0034-71672011000200017.

15. Carvalho EC, Stina APN, Marmol MT, Garbin LM, Braga FTMM, Moreli L et al. Efeito de vídeo educativo no comportamento de higiene bucal de pacientes hematológicos. Rev. Eletr. Enf. [Internet]. 2014 [acesso em: 31 dez. 2017];16(2):304-11. Disponível em: https://doi.org/10.5216/ree.v16i2.23300.

16. Stina APN, Zamarioli CM, Carvalho EC. Effect of educational video on the student's knowledge about oral hygiene of patients undergoing chemotherapy. Esc Anna Nery [Internet]. 2015 [acesso em: 31 dez. 2017];19(2):220-5. Disponível em: http://doi.org/10.5935/1414-8145.20150028.

17. Cardoso AF, Moreli L, Braga FT, Vasques Cl, Santos CB, Carvalho EC. Effect of a video on developing skills in undergraduate nursing students for the management of totally implantable central venous access ports. Nurse Educ Today [Internet]. 2012 [acesso em: 31 dez. 2017];32(6):709-13. Disponível em:

http://doi.org/10.1016/j.nedt.2011.09.012.

18. Sowan AK, Idhail JA. Evaluation of an interactive web-based nursing course with streaming videos for medication administration skills. Int J Med Inform [Internet]. 2014 [acesso em: 31 dez. 2017];83(8):592-600. Disponível em:

http://doi.org/10.1016/j.ijmedinf.2014.05.004.

19. Razera APR, Trettene AS, Mondini CCSD, Cintra FMRN, Tabaquim MLM. Vídeo educativo: estratégia de treinamento para cuidadores de crianças com fissura labiopalatina. Acta Paul Enferm [Internet]. 2016 [acesso em: 31 dez. 2017];29(4):430-8. Disponível em: http://doi.org/10.1590/1982-0194201600059.

20. Williams B, French J, Brown T. Can interprofessional education DVD simulations provide an alternative method for clinical placements in nursing? Nurse Educ Today [Internet]. 2009 [acesso em: 31 dez. 2017];29(6):666-70. Disponível em: http://doi.org/10.1016/j.nedt.2009.02.008. 